\title{
On-pump versus off-pump coronary artery bypass surgery for multi-vessel coronary revascularization
}

\author{
Alina Zubarevich ${ }^{1}$, Bakytbek Kadyraliev ${ }^{2}$, Vagram Arutyunyan ${ }^{2}$, Vahe Chragyan ${ }^{2}$, \\ Magomedganipa Askadinov², Artem Sozkov², Danil Ponomarev ${ }^{2}$, Irina Zyazeva², \\ Michel Pompeu Barros Oliveira Sá ${ }^{3}$, Anja Osswald ${ }^{1}$, Konstantinos Tsagakis ${ }^{1}$, Daniel Wendt ${ }^{1}$, \\ Arjang Ruhparwar ${ }^{1}$, Alexander Weymann ${ }^{1}$, Konstantin Zhigalov ${ }^{1}$
}

${ }^{1}$ Department of Thoracic and Cardiovascular Surgery, West German Heart and Vascular Center, University of Duisburg-Essen, Essen, Germany; ${ }^{2}$ Department of Cardiovascular Surgery, S.G. Sukhanov Federal Center of Cardiovascular Surgery, E.A. Vagner Perm State Medical University, Perm, Russian Federation; ${ }^{3}$ Division of Cardiovascular Surgery of Pronto Socorro Cardiológico de Pernambuco-PROCAPE, University of Pernambuco, Recife, Brazil

Contributions: (I) Conception and design: A Zubarevich, K Zhigalov, B Kadyraliev, A Weymann, A Ruhparwar; (II) Administrative support: A Weymann, D Wendt, A Ruhparwar, K Tsagakis; (III) Provision of study materials or patients: B Kadyraliev, V Arutyunyan, V Chragyan, M Askadinov, A Sozkov, D Ponomarev, I Zyazeva; (IV) Collection and assembly of data: B Kadyraliev, V Arutyunyan, V Chragyan, M Askadinov, A Sozkov, D Ponomarev, I Zyazeva; (V) Data analysis and interpretation: A Zubarevich, K Zhigalov, A Weymann, A Osswald, MPB Oliveira Sá, A Ruhparwar; (VI) Manuscript writing: All authors; (VII) Final approval of manuscript: All authors.

Correspondence to: Alina Zubarevich, MD. Department of Thoracic and Cardiovascular Surgery, West German Heart and Vascular Center, University of Duisburg-Essen, Essen, Germany. Email: alina.zubarevich@gmail.com.

Background: This study aims to compare the operative and postoperative results of on-pump (ONCAB) and off-pump (OPCAB) coronary artery bypass surgery (CABG) for multi-vessel coronary revascularization ( $\geq 4$ anastomoses).

Methods: From May 2018 to August 2019, a total of 120 patients $(22.5 \%$ women, mean age 61.5 \pm 8.4 years) received either ONCAB (Group 1, $\mathrm{n}=60$ ) or OPCAB (Group 2, n=60) for multi-vessel coronary artery disease (CAD). Preoperative left ventricular (LV) ejection fraction (EF) was $53.1 \% \pm 8.4 \%$. Median EuroSCORE II was 1.59 (interquartile range, 1.01-2.54). The median number of performed coronary anastomoses was 4 (interquartile range, 4-5), with equal distribution in both groups $(\mathrm{P}=0.4)$. All procedures were performed by highly experienced surgeons. The primary endpoints were overall survival at 30 days and freedom from severe adverse events (SAE), which included myocardial infarction (MI), coronary artery re-operation, and re-thoracotomy, caused by bleeding and stroke.

Results: The overall survival in both groups was $100 \%$ with no intraoperative OPCAB-to-on-pump conversion. The median procedure time was $169 \mathrm{~min}$ (interquartile range, 150-179 min) for Group 1 and $183 \mathrm{~min}$ (interquartile range, 169-205 min) for Group $2(\mathrm{P}<0.001)$. The overall freedom from SAE numbered $93.3 \%$ (98.3\% vs. 88.3\%, $\mathrm{P}=0.030$ ). Postoperative $\mathrm{MI}$ rate was $2.5 \%(\mathrm{n}=3$ ) with no significant difference for either group ( 0 vs. $5.0 \%, \mathrm{P}=0.100)$. One $\mathrm{MI}$ patient underwent a re-operation, and two other patients received a conservative treatment. A total of $2.5 \%(n=3)$ of patients underwent a re-thoracotomy on account of bleeding ( 0 vs. $5.0 \%, \mathrm{P}=0.100)$; no anastomosis-related bleeding was detected. Blood transfusion was applied in $31.7 \%$ of patients $(38.3 \%$ vs. $25.0 \%, \mathrm{P}=0.090)$. A total of $1.7 \%$ of patients $(1.7 \%$ vs. $1.7 \%$, $\mathrm{P}=0.800$ ) developed a stroke. Ventilation time, intensive care unit stay, and hospital stay were similar in both groups.

Conclusions: ONCAB showed superior freedom from SAE and shorter procedure times when compared to OPCAB for multi-vessel coronary artery revascularization. 
Keywords: Off-pump coronary artery bypass surgery (OPCAB); off-pump; coronary artery bypass surgery (CABG)

Submitted Mar 12, 2020. Accepted for publication Aug 14, 2020.

doi: $10.21037 /$ jtd-20-1284

View this article at: http://dx.doi.org/10.21037/jtd-20-1284

\section{Introduction}

Coronary artery bypass grafting (CABG) remains the most frequently performed procedure in the field of adult cardiac surgery. Even though the first attempts to perform this surgery were done on a beating heart without the cardiopulmonary bypass (CPB), nowadays the conventional CABG-procedure is mostly performed on-pump (1). Although there are several publications showing no long-term difference in the outcome after the on-pump (ONCAB) and off-pump (OPCAB) CABG procedures (2), there is still an ongoing debate on this matter. The reason for this is the controversy in the outcomes of the major coronary trials. For example, the lack of experience of the off-pump surgeons in the ROOBY trial or the low volume of the performing centers might have influenced the study outcomes. There is a wide range of complications, caused by the extracorporeal circulation and cardiac arrest (3). The negative influence of the cardioplegia on the myocardium tissue, ischemia-reperfusion damage (4), impairment of the liver (5), kidney and lung function (6), abdominal ischemia (7), stroke (8), immunosuppression and coagulopathy (9), leading to bleeding complications, systemic inflammatory response, and postoperative cardiac arrhythmia (10) are all the problems associated with the utilization of CPB. Moreover, the increasing age of the patients and the increasing patient population with impaired left ventricular function are adding to the complexity of the decision-making on the revascularization strategy. These patients tend to present more frequently with a diffuse multi-vessel coronary disease, which also complicates the revascularization and leads to early complications if operated on-pump (11). Offpump procedure allows to avoid some complications of CPB like hemodilution, embolism from manipulation on the aorta, non-pulsatile flow, and systemic inflammatory response induced by the ischemia reperfusion injury (12). The most beneficial is the off-pump procedure in high-risk patients with chronic obstructive lung disease, kidney injury, atherosclerosis, and multi-vessel coronary disease $(13,14)$.

In this study, we present a single center experience of the off-pump $v s$. on-pump multi-vessel revascularization in patients with multi-vessel coronary artery disease (CAD). We present the following article in accordance with the STROBE reporting checklist (available at http://dx.doi. org/10.21037/jtd-20-1284).

\section{Methods}

\section{Study population and design}

This study is a retrospective, observational cohort review with prospectively collected data. All patients $(\mathrm{n}=120)$ with multivessel CAD, who underwent a surgical revascularization (Group 1: on-pump n=60 and Group 2: off-pump n=60) by experienced surgeons from May 2018 to August 2019 were included in the study. Data collected prospectively as part of the institutional database included detailed information on patients' demographics and baseline clinical characteristics and their laboratory, echocardiographic, and hemodynamic parameters, as well as intraoperative variables and postoperative outcomes. The primary endpoints were overall survival after the operation at 30 days and freedom from severe adverse events (SAE), which included myocardial infarction (MI), coronary artery reoperation, and rethoracotomy caused by bleeding and stroke.

The study was conducted in accordance with the Declaration of Helsinki (as revised in 2013).

The ethical board of the institution approved the study protocol and data gathering and waived the need for informed consent form patients for data use. All patients signed the informed consent on follow-up.

To describe the angiographic atherosclerosis, we used the Gensini score, which enables one not only to quantify the degree of arterial narrowing, but also to specify its localization (15). This empirical index is based on the position, the percentage of the stenosis, and the artery where it appears.

\section{Surgical technique}

All surgeries were performed under general endotracheal anesthesia. Coronary arteries in the OPCAB procedures 

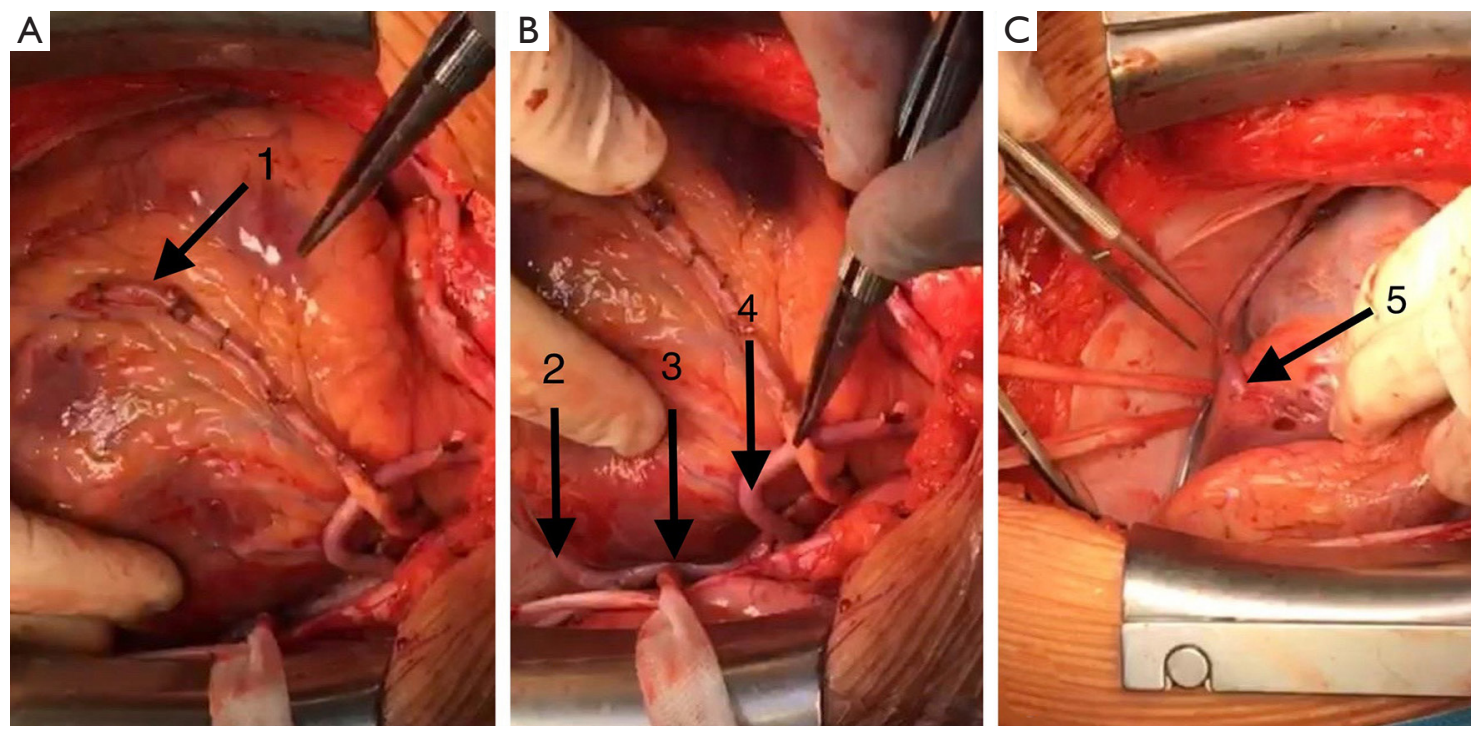

Figure 1 Intraoperative images of an OPCAB procedure with five anastomoses. (A) Left internal thoracic artery (skeletonized) to the left anterior descendent coronary artery anastomosis [1]; (B) sequential saphenous vein graft to the second marginal branch [2], first marginal branch [3], and diagonal branch [4]; (C) saphenous vein graft to the right coronary artery anastomosis [5].

were exposed with the aid of deep pericardial stitches and heart stabilizers.

Intracoronary shunts were used for myocardial protection in all off-pump cases. Proximal anastomoses were performed with a partial clamp technique.

The on-pump cases were performed under standard CPB procedure with ascending aortic cannulation, cannulation of the right atrium with a single two-staged cannula, and aortic vent. Anterograde warm blood cardioplegia (Calafiore) via aortic root was used to arrest the heart. Proximal anastomoses were performed with a partial clamp technique. In every coronary procedure, we apply epiaortic ultrasound to evaluate or map where to put the aortic clamp, the partial clamp for the proximal anastomosis, or, eventually, to decide to perform the procedure in the single clamp technique if the partial clamp is impossible to place. The grafts used were left internal mammary artery as well as saphenous vein grafts (SVGs) (Figure 1). The total mean number of bypasses was 4 [interquartile range (IQR), 4-5] per patient (maximum 7). Left internal mammary was anastomosed either as a single graft or sequentially to LAD and diagonal branch. SVG was anastomosed sequentially or as a single graft when anastomosed to the proximal right coronary artery (RCA). In all the cases, complete revascularization was achieved. The indication for each procedure was made in accordance with the current guidelines (16).

\section{Variables and definitions}

Respiratory complications were re-intubation, postoperative tracheotomy and a prolonged ventilation time for more than a week postoperatively. Postoperative MI was defined as any or all of a troponin elevation beyond the normal upper limit, as defined by the testing laboratory, without electrocardiographic (ECG) changes, or clinical symptoms (severe chest pain or radiotherapy to the left arm or jaw), or ECG changes consistent with MI. Stroke was defined as a persistent (lasting longer than 1 week) neurologic deficit, consistent with a central nervous system lesion that occurred within 30 days of operation.

\section{Statistical analysis}

The data were analyzed using IBM SPSS Version 25 (IBM Corporation, Chicago, Ill., USA). We used the Kolmogorov-Smirnov test to prove the data for normal distribution. Quantitative data are expressed as the mean and standard deviation (SD) for normally distributed variables and as the median and IQR for not normally distributed variables. Categorical data are expressed as frequency and percentage. The significance of survival differences between the groups was assessed with Log-Rank and Breslow tests. A value of $\mathrm{P}<0.05$ was considered to be statistically significant. 
Table 1 Baseline characteristics and risk factors

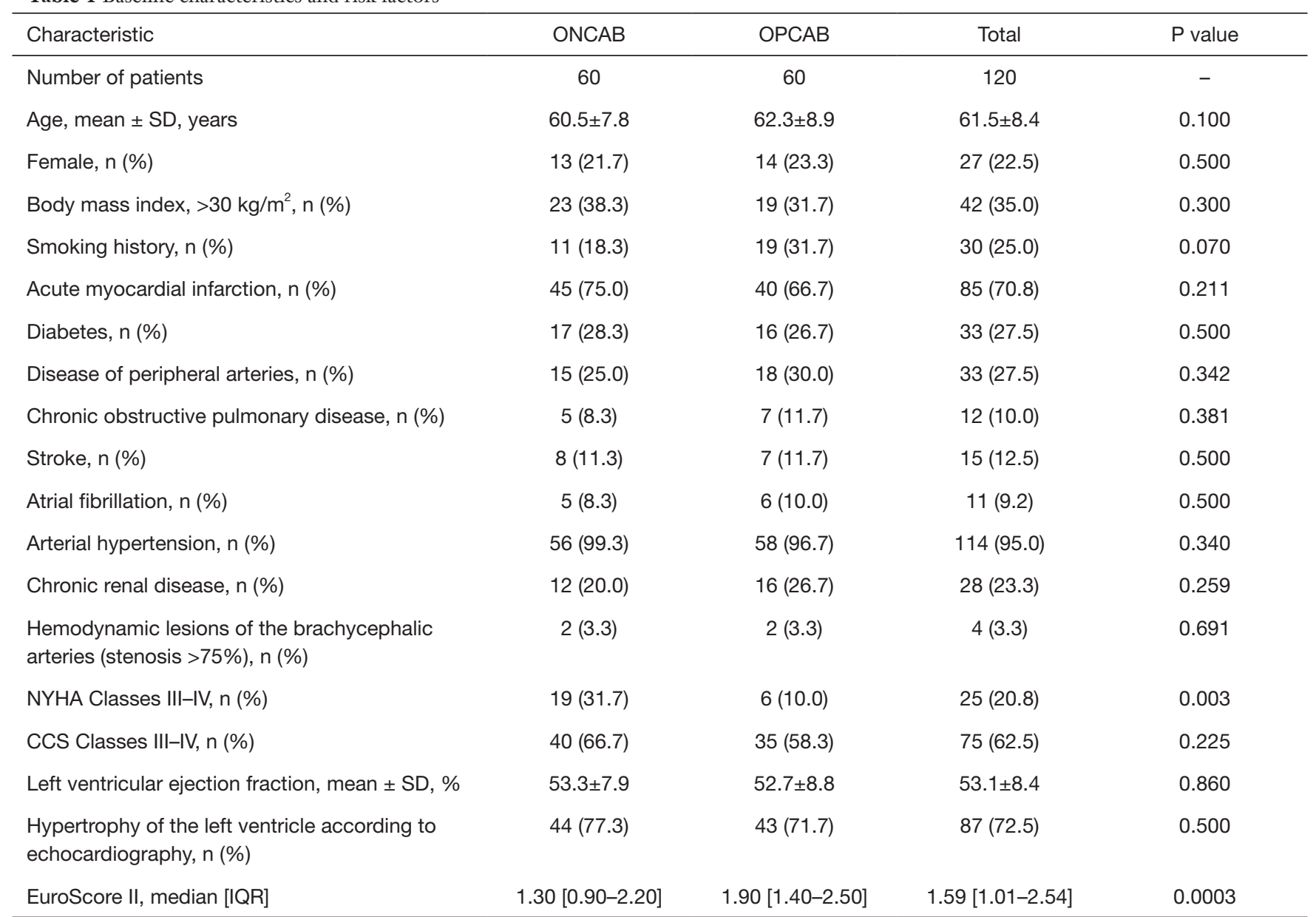

ONCAB, on-pump coronary artery bypass surgery; OPCAB, off-pump coronary artery bypass surgery; SD, standard deviation; IQR, interquartile range; CCS, Canadian Cardiovascular Society Score; NYHA, New York Heart Association Functional Classification.

\section{Results}

\section{Baseline characteristics}

From May 2018 to August 2019, a total of 120 patients (22.5\% women, mean age $61.5 \pm 8.4$ years) underwent either off-pump CABG, or on-pump CABG for multivessel CAD. There were several significant differences in the baseline characteristics between the study groups: in the on-pump group, there were significantly more patients with NYHA Class III-IV (31.7\%) vs. $10.0 \%$ in the OPCAB group $(\mathrm{P}=0.003)$, but the EuroScore II Class was significantly higher in the OPCAB group 1.90 (IQR, 1.402.50) vs. on-pump group 1.30 (IQR, 0.90-2.20) $(\mathrm{P}=0.0003)$. Other baseline characteristics were similar in both groups (Table 1).

\section{Intraoperative data}

There was no statistically significant difference in the number of grafts between two groups. In both groups, the patients received four and more bypasses (maximum seven). The operation time was longer in the OPCAB group (median $183 \mathrm{~min}$; IQR, 169-205 min) vs. median $169 \mathrm{~min}$; IQR, 150-179 min in the on-pump group $(\mathrm{P}<0.001)$. The mean time on the CPB in the on-pump groups was median $65.5 \mathrm{~min}$; IQR, 58.0-72.7 $\mathrm{min}$ (Table 2).

\section{Survival data and adverse events}

There was no difference in the survival rates at 30 days; the overall survival was $100 \%$. There was no intraoperative 
Table 2 Intraoperative data

\begin{tabular}{lcccc}
\hline Characteristic & ONCAB & OPCAB & Total & P value \\
\hline Number of grafts, median [IQR] & $4[4-5]$ & $4[4-4]$ & $4[4-5]$ & 0.4 \\
Operation time, median [IQR], min & $169[150-179]$ & $183[169-205]$ & $175[160-195]$ & $<0.001$ \\
CPB time, median [IQR], min & $65.5[58.0-72.7]$ & - & $65.5[58.0-72.7]$ & -
\end{tabular}

ONCAB, on-pump coronary artery bypass surgery; OPCAB, off-pump coronary artery bypass surgery; CPB, cardiopulmonary bypass; $I Q R$, interquartile range.

Table 3 Postoperative outcomes

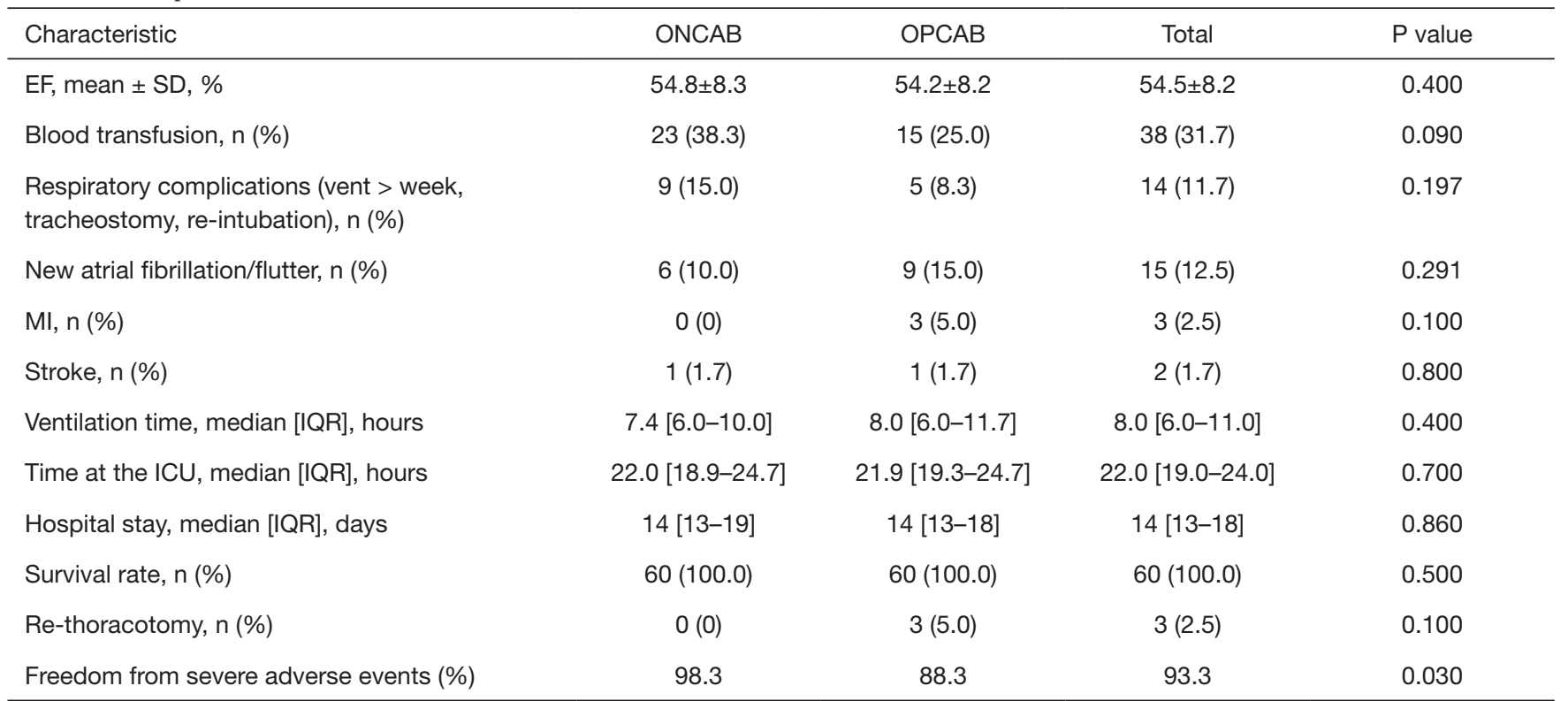

ONCAB, on-pump coronary artery bypass surgery; OPCAB, off-pump coronary artery bypass surgery; EF, ejection fraction; SD, standard deviation; IQR, interquartile range; ICU, intensive care unit.

OPCAB-to-ONCAB conversion. The overall freedom from SAE numbered $93.3 \%$ (98.3\% vs. $88.3 \%$, $\mathrm{P}=0.030$ ) with higher SAE rate in the off-pump group. Postoperative MI rate was $2.5 \%(n=3)$ with no significant difference for either group ( 0 vs. $5.0 \%, \mathrm{P}=0.100)$. One MI patient underwent a redo procedure, and two other patients received a conservative treatment. Some $2.5 \%$ $(n=3)$ of patients underwent a re-thoracotomy on account of bleeding ( 0 vs. $5.0 \%, \mathrm{P}=0.100)$; no anastomosis-related bleeding was detected. Blood transfusion was applied in $31.7 \%$ of patients $(38.3 \%$ vs. $25.0 \%, \mathrm{P}=0.090)$. A total of $1.7 \%$ of patients $(1.7 \%$ vs. $1.7 \%, \mathrm{P}=0.800)$ developed a stroke.

Furthermore, there was no significant difference in the time spent at the intensive care unit (ICU) (median 22.0 days; IQR, 18.9-24.4 days on-pump vs. median 21.9 days;
IQR, 19.3-24.7 days off-pump) and the ventilation time between the groups receiving on-pump (median 7.4 hours; IQR, 6.0-10.0 hours) and OPCAB (median 8.0 hours; IQR, 6.0-11.7 hours) revascularization (Table 3).

\section{Discussion}

CABG remains the most frequent performed procedure in the field of adult cardiac surgery. In this study, we compared 120 patients with multi-vessel CAD receiving a surgical coronary revascularization on-pump and OPCAB. As described, there is a large number of complications associated with the CPB. Comparing the two groups of patients, we were expecting the lower rates of $\mathrm{CPB}$ associated adverse events in the OPCAB group. In our study, we could not show that patients in the OPCAB 
group required fewer blood transfusions than the patients in the on-pump group ( $38.3 \%$ vs. $25.0 \%, \mathrm{P}=0.090)$. We also did not see any significant difference in the respiratory complications between the groups $(8.3 \%$ vs. $15.0 \%$, $\mathrm{P}=0.197)$. Even though the patients in the on-pump group presented with a higher NYHA Class (NYHA III-IV 31.7\% in the on-pump group vs. $10.0 \%$ in the OPCAB group), the patients in the OPCAB group had higher EuroScore II rates, which means they carry a higher postoperative risk. We should also mention that the higher Euro-Score rates in the OPCAB presented in our study were still $<2 \%$, which is considered to be in the low-risk range (17). Despite the higher EuroScore Class in the OPCAB cohort, there was no difference in the postoperative survival between the groups: the overall 30 -day survival was $100 \%$, which shows that the $\mathrm{OPCAB}$ procedure is safe and non-inferior in mortality for these groups of patients. There is a controversial opinion that the OPCAB procedure might be associated with an incomplete revascularization and a tendency on the part of surgeons to perform fewer anastomoses than in on-pump procedure (18). Our study presents both groups with the same number of anastomoses (4-5, max. 7) in patients with multi-vessel CAD with comparable complexity of CAD defined by Gensini score.

There is an ongoing conversation about the noninferiority of the OPCAB procedure to the on-pump revascularization. The interest in the $\mathrm{OPCAB}$ surgery arose again in the 90th to reduce the complications caused by $\mathrm{CPB}$. A large number of studies on this matter have been published, but there is still no definitive answer to the question whether the OPCAB surgery is non-inferior to the conventional type of the coronary revascularization. In the ROOBY trial with 2,203 randomized patients, Shroyer et al. describes better survival and better graft patency in the onpump group at 5 years (19). A question about the surgeons' experience participating in the trial was raised immediately. A median number of off-pump cases performed by the surgeons participating in the ROOBY trial was 50, which means that a significant number of surgeons were still in the learning curve for the OPCAB procedure. In our study, all the procedures were performed by experienced surgeons performing over 125 CABG procedures (OPCAB and onpump) per year. Another large trial-the CORONARY trial-comparing both procedures on 4,752 randomized patients showed no significant difference between offpump and on-pump CABG with respect to the 30-day rate of death, MI, stroke, or renal failure requiring dialysis. The same conclusion followed after the 5-year follow- up of the CORONARY trial was completed (2). Carmona et al. performed a propensity score matching analysis for over 2,000 patients and reported no significant difference in mortality, but found lower incidence of postoperative pulmonary, neurological, and renal complications in the OPCAB group, which showed that OPCAB procedure is safe for the patients and is non-inferior in terms of mortality and morbidity compared to the on-pump CABG. This propensity score matching analysis also described the lower number of the distal anastomoses and anastomoses to the left lateral wall in the OPCAB group, which we did not see in our study. All the surgeons in this trial completed a sufficient OPCAB training (20). Diegeler et al. came to the same conclusion in the largest German GOPCABE trial with 2,539 patients in high-volume OPCAB institutions. The trial revealed no significant difference in the composite end point consisting of death, stroke, MI, new renal replacement therapy, or repeat revascularization at 30 days and 1 year. Although there was no significant difference in the survival, the study confirmed shorter periods of ICU and hospital stay, as well as reduced need for blood transfusion for the OPCAB patients, which was also confirmed in our results. All the procedures were performed by highly qualified coronary surgeons (21). Similar results were reported by Kirmani et al. in the largest single center propensity score matching study, with more than 5,000 patients per group (22). The study also emphasized the safety of teaching and performing coronary revascularization OPCAB, because of the low conversion rate to the on-pump procedure and clinically comparable number of performed anastomoses in both groups, as also described in our study.

In this review, we report on significantly longer operative times in the OPCAB group (183 vs. 169 min, $\mathrm{P}<0.001)$ with the similar number of anastomoses in both groups (4-5, maximum 7). Though the operative time is known to be an independent negative predicting factor of mortality during a cardiac procedure, because of the completeness of the multi-vessel revascularization and a number of peripheral anastomoses in the $\mathrm{OPCAB}$ group, the operative time had no influence on the mortality. Though we did not see any difference in the mortality at 30 days, the SAE rate was-at $11.7 \%$ - significantly higher in the off-pump group than in the on-pump patients $(1.7 \%, \mathrm{P}=0.030)$.

\section{Study limitations}

We have to report on several study limitations. First, alongside with the observational type of study, we were 
limited by the number of patients. Second, the assignment to one of the study groups was done via surgeons' decision on the type of CABG. Finally, the graft patency was not angiographically assessed. Instead, the rate of repeated revascularization was used as a clinical correlation parameter.

\section{Conclusions}

In conclusion, in our study, we aimed to show the noninferiority of the OPCAB method in the multi-vessel coronary revascularization. Even though the SAE rates and the operative time in the OPCAB group were significantly higher than in the on-pump group, we could not report any significant difference in the mortality between the groups. We also detected no signs of an incomplete revascularization in the OPCAB group. Despite our findings, like the major coronary trials, our study portrays the OPCAB method of the coronary multi-vessel revascularization safe and equally effective as a conventional on-pump revascularization.

\section{Acknowledgments}

Funding: None.

\section{Footnote}

Reporting Checklist: The authors have completed the STROBE reporting checklist. Available at http://dx.doi. org/10.21037/jtd-20-1284

Data Sharing Statement: Available at http://dx.doi. org/10.21037/jtd-20-1284

Conflicts of Interest: All authors have completed the ICMJE uniform disclosure form (available at http://dx.doi. org/10.21037/jtd-20-1284). The authors have no conflicts of interest to declare.

Ethical Statement: The authors are accountable for all aspects of the work in ensuring that questions related to the accuracy or integrity of any part of the work are appropriately investigated and resolved. The study was conducted in accordance with the Declaration of Helsinki (as revised in 2013). The ethical board of the institution approved the study protocol (NO.: 2322) and data gathering and waived the need for informed consent form patients for data use. All patients signed the informed consent on follow-up.

Open Access Statement: This is an Open Access article distributed in accordance with the Creative Commons Attribution-NonCommercial-NoDerivs 4.0 International License (CC BY-NC-ND 4.0), which permits the noncommercial replication and distribution of the article with the strict proviso that no changes or edits are made and the original work is properly cited (including links to both the formal publication through the relevant DOI and the license). See: https://creativecommons.org/licenses/by-nc-nd/4.0/.

\section{References}

1. Adams DH, Chikwe J. On-Pump CABG in 2018: Still the Gold Standard. J Am Coll Cardiol 2018;71:992-3.

2. Lamy A, Devereaux PJ, Prabhakaran D, et al. Five-Year Outcomes after Off-Pump or On-Pump Coronary-Artery Bypass Grafting. N Engl J Med 2016;375:2359-68.

3. Salis S, Mazzanti VV, Merli G, et al. Cardiopulmonary bypass duration is an independent predictor of morbidity and mortality after cardiac surgery. J Cardiothorac Vasc Anesth 2008;22:814-22.

4. Chen S, Hua F, Lu J, et al. Effect of dexmedetomidine on myocardial ischemia-reperfusion injury. Int J Clin Exp Med 2015;8:21166-72.

5. Duan L, Hu G, Jiang $M$, et al. Postoperative abnormal liver function in children with heart surgery. Zhong Nan Da Xue Xue Bao Yi Xue Ban 2018;43:1007-13.

6. Pickering JW, James MT, Palmer SC. Acute kidney injury and prognosis after cardiopulmonary bypass: a meta-analysis of cohort studies. Am J Kidney Dis 2015;65:283-93.

7. Dong G, Liu C, Xu B, et al. Postoperative abdominal complications after cardiopulmonary bypass. J Cardiothorac Surg 2012;7:108.

8. Rankin JM, Silbert PL, Yadava OP, et al. Mechanism of stroke complicating cardiopulmonary bypass surgery. Aust N Z J Med 1994;24:154-60.

9. Romlin BS, Wahlander H, Synnergren M, et al. Earlier detection of coagulopathy with thromboelastometry during pediatric cardiac surgery: a prospective observational study. Paediatr Anaesth 2013;23:222-7.

10. Sandler N, Kaczmarek E, Itagaki K, et al. Mitochondrial DAMPs Are Released During Cardiopulmonary Bypass Surgery and Are Associated With Postoperative Atrial Fibrillation. Heart Lung Circ 2018;27:122-9.

11. Arbel Y, Fuster V, Baber U, et al. Incidence, determinants 
and impact of acute kidney injury in patients with diabetes mellitus and multivessel disease undergoing coronary revascularization: Results from the FREEDOM trial. Int J Cardiol 2019;293:197-202.

12. Trehan N, Mishra M, Sharma OP, et al. Further reduction in stroke after off-pump coronary artery bypass grafting: a 10-year experience. Ann Thorac Surg 2001;72:S1026-32.

13. Dhurandhar V, Saxena A, Parikh R, et al. Outcomes of On-Pump versus Off-Pump Coronary Artery Bypass Graft Surgery in the High Risk (AusSCORE > 5). Heart Lung Circ 2015;24:1216-24.

14. Enginoev S, Koz'min D, Magomedov G, et al. Ligation of Left Atrial Appendage during Off-Pump Coronary Surgery. Thorac Cardiovasc Surg 2019. [Epub ahead of print]. doi:10.1055/s-0039-1695758.

15. Gensini GG. A more meaningful scoring system for determining the severity of coronary heart disease. Am J Cardiol 1983;51:606.

16. Claeys MJ, Vandekerckhove Y, Cosyns B, et al. Summary of 2019 ESC Guidelines on chronic coronary syndromes, acute pulmonary embolism, supraventricular tachycardia and dislipidaemias. Acta Cardiol 2020. [Epub ahead of print]. doi: 10.1080/00015385.2019.1699282.

Cite this article as: Zubarevich A, Kadyraliev B, Arutyunyan V, Chragyan V, Askadinov M, Sozkov A, Ponomarev D, Zyazeva I, Oliveira Sá MPB, Osswald A, Tsagakis K, Wendt D, Ruhparwar A, Weymann A, Zhigalov K. On-pump versus offpump coronary artery bypass surgery for multi-vessel coronary revascularization. J Thorac Dis 2020;12(10):5639-5646. doi: $10.21037 /$ jtd-20-1284
17. Borracci RA, Rubio M, Baldi JJ, et al. Mortality in lowand very low-risk patients undergoing cardiac surgery: evaluation according to the EuroSCORE II as a new standard. Cardiol J 2015;22:495-500.

18. Robertson MW, Buth KJ, Stewart KM, et al. Complete revascularization is compromised in off-pump coronary artery bypass grafting. J Thorac Cardiovasc Surg 2013;145:992-8.

19. Shroyer AL, Hattler B, Wagner TH, et al. Five-Year Outcomes after On-Pump and Off-Pump CoronaryArtery Bypass. N Engl J Med 2017;377:623-32.

20. Carmona P, Paredes F, Mateo E, et al. Is off-pump technique a safer procedure for coronary revascularization? A propensity score analysis of 20 years of experience. Interact Cardiovasc Thorac Surg 2016;22:612-8.

21. Diegeler A, Borgermann J, Kappert U, et al. FiveYear Outcome After Off-Pump or On-Pump Coronary Artery Bypass Grafting in Elderly Patients. Circulation 2019;139:1865-71.

22. Kirmani BH, Holmes MV, Muir AD. Long-Term Survival and Freedom From Reintervention After Off-Pump Coronary Artery Bypass Grafting: A Propensity-Matched Study. Circulation 2016;134:1209-20. 